\title{
Le cas des thèses d'ingénieur-docteur à Lyon : Une nouvelle façon de penser l'enseignement et la recherche en chimie dans l'entre-deux-guerres
}

\author{
Virginie FONTENEAU*
}

\section{Résumé}

Par l'étude des thèses d'ingénieur-docteur soutenues en chimie à Lyon pendant l'entre-deux guerres, cet article a deux objectifs. Le premier est de montrer l'intrication des institutions de formation en chimie sur un territoire donné. Loin de l'image d'institutions cloisonnées et autonomes que les monographies peuvent produire, le cas lyonnais est particulièrement éclairant sur la mise en commun de professeurs, d'étudiants et de locaux entre institutions qu'elles soient vouées à la formation des scientifiques ou des ingénieurs, qu'elles soient académiques ou non, publiques ou privées. Le deuxième objectif est de poser la question de la place de la chimie industrielle par rapport à la chimie dite pure. Ainsi, au niveau de la formation doctorale, cette étude permettra de démontrer que se côtoient au sein des laboratoires des étudiants préparant des thèses d'ingénieur-docteur, des thèses d'université ainsi que des thèses d'État. Les différences entre ces thèses sont plus complexes que la ligne de partage encore trop communément tracée entre science/université et industrie/ingénieur ne le laisserait supposer. Cette étude permet donc d'apporter également un nouvel éclairage aux relations entre ces deux pôles en chimie.

\section{Mots-clés}

Chimie. Ingénieurs. Facultés des sciences. Thèses. Entre-deuxguerres. Offre locale d'enseignement scientifique et technique.

\begin{abstract}
Our study of the theses written by Engineer-Phd (ingénieur-docteur) students in chemistry in Lyons during the interwar period leads us to two major conclusions that we present here. First, the ways in which the training institutions in chemistry are interconnected across a given territory, which undermines the image of compartmentalized and autonomous institutions usually presented by monographs. The case of Lyons provides an interesting

\footnotetext{
Virginie FONTENEAU, GHDSO-EST-EA 1610, Université Paris Sud/ Paris Saclay - Groupe d'histoire de la chimie.
} 
example where we can observe a pool of teachers, students and locations that are shared between local institutions including academic or nonacademic actors, private or public resources, employed in the training of scientists or engineers. The second element concerns the relationship between industrial chemistry and the so-called 'pure' chemistry. Our study of the students, their laboratories and the scientific contents of their theses, shows that students (academics and engineers) who prepared engineerdoctor theses, university theses (thèses d'université) and doctoral theses (thèses d'État) worked together and interacted within the chemical laboratories of the faculty of science. The differences between these three types of theses are more complex than one would imagine based on the commonly used distinction between science/university and industry/engineer would suggest. This study also helps to provide new insights concerning the relationship between these two poles in chemistry.

\section{Keywords}

Chemistry. Engineers. Faculty of science. Thesis. Interwar. Local supply of scientific and technical education.

\section{Introduction}

Les origines et la portée du titre d'ingénieur-docteur sont encore peu connues. Les thèses d'ingénieur-docteur produites dans l'entre-deux-guerres ne font pas partie des corpus généralement étudiés lorsque sont faites des études sur ce qu'est la recherche dans cette période. J'expliquerai donc tout d'abord la genèse de ce titre et je montrerai que, dans cette période, s'il ne rencontre pas un succès rapide, ce n'est pas un objet marginal. Au contraire, ce titre est d'un grand intérêt dans l'étude du développement de l'enseignement supérieur en province et doit être mis en rapport avec l'émergence des instituts de faculté et le développement de deux disciplines spécifiques : la chimie et l'électrotechnique ${ }^{1}$.

\footnotetext{
${ }^{1}$ Grelon André, «Les universités et la formation des ingénieurs en France (18701914) », Formation Emploi, 27-28, 65-88 (1989).
} 
Je développerai ici le cas de la chimie dans un territoire donné - la ville de Lyon - en mobilisant l'approche préconisée par Renaud d'Enfert de travailler l'offre locale d'un point de vue disciplinaire ${ }^{2}$, ce que, dans le cadre de cet article, je ferai sur l'enseignement supérieur et la recherche scientifique ou technique. Le cas de Lyon a déjà été travaillé pour ce qui est des relations science/industrie par Terry $\mathrm{Shinn}^{3}$ et Mary Jo $\mathrm{Nye}^{4}$, qui ont pointé l'importance des formations pour l'industrie. Pour Terry Shinn, cette intervention industrielle a un effet négatif sur la recherche fondamentale. Pour Mary Jo Nye, le développement des formations en chimie vers l'industrie est positif pour la science à Lyon au $\mathrm{XIX}^{\mathrm{e}}$ siècle et jusqu'à la Première guerre mondiale. À la fin de la Première guerre mondiale, il y a un renversement de tendance, Lyon serait submergé par les étudiants souhaitant se spécialiser pour l'industrie, brisant un équilibre ${ }^{5}$. Cet afflux provoquerait le départ des étudiants ambitieux vers Paris, où ils pourraient recevoir une formation de science pure, plus valorisante ${ }^{6}$.

2 D'Enfert Renaud, Pour une histoire "par en bas» de l'enseignement mathématique (XIX $-X X^{e}$ siècles), Mémoire pour l'obtention de l'habilitation à diriger des recherches, Université Paris Sud, 2012.

${ }^{3}$ Shinn Terry, «The French science faculty system, 1808-1914: Institutional change and research potential in mathematics and the physical sciences ", Historical Studies in the Physical Sciences, 10, 271-332 (1979).

${ }^{4}$ Nye Mary Jo, Science in the Provinces : scientific communities and provincial leadership in France, 1860-1930, University of California Press, Berkeley, 1986.

${ }^{5}$ Nye M. J., Science in the Provinces, op. cit., note 4, p. 193 : « Provincial universities such as Lyon, which long had been offering vocational and specialized scientific and engineering education, were now inundated with increasing numbers of students oriented toward specialized, professional training. For those, like Grignard, whose activities in earlier years had moved back and forth between research interests and practical education, equilibrium was lost $»$.

${ }^{6}$ Nye M. J., Science in the Provinces, op. cit., note 4, p. 193. 
Dans les deux cas, les seules institutions étudiées sont les facultés des sciences, et les acteurs sont les titulaires de chair.

Je présenterai donc le système local d'enseignement supérieur ${ }^{7}$ pour la chimie, revenant sur des points de son développement nécessaires pour appréhender les enjeux du titre d'ingénieur-chimiste à Lyon. Dans une première partie, je ferai une étude des thèses d'ingénieur-docteur et dans une seconde partie, je les positionnerai dans un ensemble plus vaste constitué des thèses d'université et des thèses d'État.

\section{La genèse du titre d'ingénieur-docteur}

Le titre d'ingénieur-docteur est créé par décret du 30 avril 1923. Tous les ingénieurs ne peuvent y prétendre, la liste des écoles autorisées ne paraît qu'un an plus tard. Le diplôme est délivré après deux années passées dans un laboratoire de faculté des sciences et après présentation d'un « travail technique reconnu satisfaisant par un jury d'examen composé des membres de la faculté $)^{8}$. Avec ces deux conditions assez vagues en fait, apparaît l'enjeu explicite de ce nouveau titre, il s'agit de «former des techniciens à l'esprit

\footnotetext{
${ }^{7}$ Grossetti Michel (dir.) Université et territoire, un système local d'enseignement supérieur, Toulouse et Midi-Pyrénées, Presses universitaires du Mirail, Toulouse, 1994. Rollet Laurent, "Peut-on faire l'histoire des pôles scientifiques ? ", Histoire de l'éducation, 122, 95-113 (2009).

8 Archives nationales de France (AN), F17/17844. Conseil supérieur de l'enseignement technique. Procès-verbaux des réunions et projets de textes, 19221938.
} 
scientifique $»^{9}$, c'est-à-dire à la recherche, et la faculté serait un lieu légitime de formation par opposition aux écoles d'ingénieurs ${ }^{10}$.

Il y a déjà eu précédemment une ouverture de la formation doctorale mais non explicitement tournée vers les ingénieurs. Gabriel Lippmann (1845-1921), docteur ès sciences physiques et professeur de physique à la faculté des sciences de Paris, propose la création d'un doctorat d'université, pour lequel la licence n'est pas exigible. Il suffit d'obtenir deux certificats d'études supérieures ou un diplôme équivalent. Le titre, créé en 1897, permet l'accès à une formation doctorale pour des étudiants étrangers, et pour des étudiants qui ne sont pas passés par le lycée et ne peuvent prétendre à l'obtention d'une licence. Comme le doctorat d'université, le titre de docteuringénieur ne permet pas l'accès au statut de maître de conférence ni de professeur d'université.

Il y a un enjeu qui n'est pas formulé explicitement par les concepteurs du projet. Ceux-ci sont issus de l'université, le plus actif est Gabriel Koenigs (1858-1931) ${ }^{11}$, alors membre de l'Académie des sciences, professeur de mécanique physique expérimentale à la faculté des sciences de Paris. Il présente à la fin de l'année 1922 un projet approuvé par seize facultés des sciences sur dix-sept ${ }^{12}$ au conseil

\footnotetext{
9 AN, F17/17844. Procès-verbal de la réunion du Conseil supérieur de l'enseignement technique du 28 novembre 1922.

${ }^{10}$ Sur la question des ingénieurs voir: Grelon André (dir.), Les Ingénieurs de la crise: titre et profession entre les deux guerres, Éditions de l'École des hautes études en sciences sociales, Paris, 1986.

${ }^{11}$ Gispert, Hélène, « Koenigs Gabriel (1858-1931), professeur de mécanique (19231931)», in Fontanon Claudine, Grelon André (dir), Les professeurs $d u$ Conservatoire national des arts et métiers, INRP, Paris, 1994, t.1, p. 731-744.

${ }^{12}$ Il n'apparaît pas dans les archives le nom de la faculté des sciences qui n'a pas approuvé ce projet.
} 
supérieur de l'enseignement technique. Comme après la première guerre mondiale, les laboratoires des facultés des sciences souffrent du manque d'étudiants et du manque de moyens financiers, c'est un moyen d'obtenir des crédits pour équiper les laboratoires sélectionnés, et de faire venir des ingénieurs sur les bancs de la faculté ${ }^{13}$.

De ce côté-là, l'idée n'est pas tout à fait nouvelle, mais cela se jouait alors uniquement à Paris entre l'École centrale des arts et manufactures et la faculté des sciences de Paris. Le décret du 9 janvier 1917 établit pour les élèves de l'École centrale l'équivalence entre le certificat d'admission et le CES de mathématiques générales. Quatre ans plus tard, une concertation entre le recteur Paul Appell, et Léon Guillet, directeur de l'École, aboutit à l'octroi d'un deuxième certificat au choix ${ }^{14}$ aux titulaires du titre d'ingénieur des arts et manufactures pour les inciter à venir à la faculté des sciences suivre les cours pour l'obtention d'un troisième certificat ${ }^{15}$. Pour Appell, il s'agit de favoriser les échanges d'étudiants entre les deux institutions, mais ce qui est un droit d'un côté se limite à une possibilité dans l'autre sens, puisque, sur proposition du conseil de l'École, certains licenciés de sciences ${ }^{16}$ pourraient entrer en seconde année de l'École centrale.

\footnotetext{
${ }^{13}$ Pour le titre d'ingénieur-docteur, il y a obligation de mener les recherches dans un laboratoire de faculté des sciences, ce qui n'est pas le cas pour le doctorat d'université.

${ }^{14}$ Choix limité aux certificats de mécanique physique, électricité générale et chimie appliquée.

${ }^{15}$ Les élèves ingénieurs passaient ce certificat sans suivre les cours à la faculté des sciences.

${ }^{16}$ Les titulaires des certificats de calcul différentiel et calcul intégral, mécanique rationnelle, physique générale et chimie générale.
} 
Peu après le décret du 30 avril 1923, l'équivalence des certificats est abrogée ${ }^{17}$. Par rapport à l'accord parisien, le nouveau titre d'ingénieur-docteur s'appuie sur une réflexion de l'ensemble des facultés des sciences et, point crucial, fait entrer dans le dispositif les instituts techniques de faculté des sciences qui se sont créés en province à partir des années 1880. Il s'agit donc d'un élargissement géographique mais aussi d'un élargissement social du dispositif, puisque les instituts techniques ne recrutent pas sur des critères élitistes comme l'École centrale, mais sélectionnent aussi des élèves passés par le système d'enseignement primaire supérieur. Cette position est clairement exprimée par Koenigs et Dubosq dans une lettre adressée au Conseil supérieur de 1'Instruction publique en $1922^{18}$ :

"Oui, les Facultés désirent ouvrir leurs portes aux Ingénieurs, à la condition toutefois que ce ne soit pas un geste vain et de pure forme et à la condition aussi qu'il ne constitue pas un privilège pour quelques favorisés. Il est souverainement injuste qu'un jeune homme courageux au travail, vif d'intelligence, traîne toute sa vie le boulet de son origine modeste, et sous prétexte qu'il n'aura pas suivi la filière ordinaire, se voir interdire à tout jamais l'accès de la Science ".

Ce positionnement va tout à fait dans le sens de ce qui se fait à Lyon depuis le $\mathrm{XIX}^{\mathrm{e}}$ siècle comme je vais le montrer à présent.

\footnotetext{
${ }^{17}$ Journal Officiel du 29 juillet 1923, p. 7443.

${ }^{18}$ AN, F17/17890. Diplômes d'ingénieurs. Ingénieur-chimiste, ingénieur-conseil, ingénieur-commercial, ingénieur-docteur, ingénieur-électricien, ingénieur des poids et mesures: législation des diplômes, rapports, organisation des études, documentation, correspondance. 1922-1946.
} 


\section{Les formations supérieures de chimie à Lyon pendant la période de l'entre-deux-guerres}

Pour comprendre le système de formation supérieure en chimie à Lyon, il nous faut remonter au XIX ${ }^{\mathrm{e}}$ siècle. L'année 1876 est une année clé. Le ministère de l'Instruction publique décide la création de deux chaires de chimie appliquée à l'agriculture et à l'industrie pour les facultés des sciences de Lille et de Lyon. Jules Raulin (1836-1896), élève de l'École normale supérieure et assistant de Louis Pasteur pendant la mission sur la maladie des vers à soie durant les années 1869-1872, est recruté à Lyon. Il installe dès sa première année un petit laboratoire où il accueille des étudiants, qui ne viennent pas de l'université. Ce sont des élèves de l'École de la Martinière, officiellement fondée à Lyon en 1831 (avant la réouverture de la faculté des sciences en 1833) pour former de jeunes élèves entre autres à la chimie. Jules Raulin va plus loin en 1883, en saisissant l'opportunité offerte par l'emménagement de la faculté dans de vastes locaux, pour proposer une école de chimie qu'il soumet à l'approbation du ministère de l'Instruction publique et de la Chambre de commerce de Lyon. L'École de chimie industrielle de Lyon (ECIL) répond aux conditions émises par la Chambre de commerce, elle est accessible aux classes peu aisées par un système de bourses, et il y a articulation entre un haut niveau théorique et une composante appliquée. Le recrutement ne se fait pas sur diplôme et les élèves sortis en premier de l'École de la Martinière sont prioritaires. De façon très novatrice pour la France, les étudiants passent six à sept heures par jour à la paillasse pendant deux ans et sont donc formés à la pratique. Il y a donc bel et bien apparition d'un cursus informel, une 
filière au sens de Jean-Michel Chapoulie ${ }^{19}$, qui va former des chimistes académiques et industriels ${ }^{20}$. Cette école est financée à la fois par l'État (salaires du personnel, subvention pour le laboratoire et les locaux), la Chambre de commerce, ainsi que la mairie et des industriels.

En 1900, elle s'installe dans les locaux du nouvel institut de chimie de l'université de Lyon, dont la construction a été motivée par la mise en place du certificat d'études physiques, chimiques et naturelles $(\mathrm{PCN})^{21}$ et qui permet une mutualisation des différents services de chimie. À la fin de la Première guerre mondiale, l'école devient le 26 décembre 1919 une société anonyme sous le patronage de l'université de Lyon et de la Chambre de commerce de Lyon. Cette transformation achève un processus qui a débuté en juin 1918 par la signature d'une convention entre l'université de Lyon et la Société anonyme de l'École de chimie industrielle en formation, qui comprend un bail de 18 ans pour la mise à disposition de locaux. Statutairement, l'école est liée à l'université par l'immatriculation des élèves et par la délivrance des titres et diplômes.

\footnotetext{
${ }^{19}$ Chapoulie Jean-Michel, L'École d'État conquiert la France. Deux siècles de politique scolaire, PUR, Rennes, 2010.

${ }^{20}$ Sur ce sujet, voir ma communication intitulée « L'offre locale d'enseignement : la chimie à Lyon au $\mathrm{XIX}^{\mathrm{e}}$ siècle» au séminaire L'offre locale d'enseignement scientifique et technique : approches disciplinaires (XIX $-X X^{e}$ siècles), organisé par Renaud d'Enfert et Virginie Fonteneau du Groupe d'histoire et de diffusion des sciences d'Orsay (GHDSO, EST-EA 1610) dans le cadre du LIDEX « Interactions between science, innovation and society de l'Université Paris Saclay », le 23 juin 2015. Un article est en préparation.

${ }^{21}$ Ce certificat institué par décret du 31 juillet 1893 est un enseignement obligatoire délivré par les facultés des sciences et dont l'obtention est obligatoire pour les étudiants souhaitant s'inscrire en faculté de médecine.
} 
Sous l'angle de l'institution, cette transformation peut apparaître comme un éloignement de la faculté des sciences alors qu'il n'en est rien, car ce statut formalise une hybridation déjà présente dans le projet de Jules Raulin. L'intrication des deux structures se comprend aussi lorsque l'on s'intéresse au personnel enseignant. Victor Grignard (1871-1935) en devient directeur en 1921, il est alors professeur de chimie générale à la faculté des sciences, et le restera jusqu'à son décès. La liste du personnel en 1929 montre que la structure enseignante s'appuie beaucoup sur les ressources de la faculté : son directeur adjoint est Louis Meunier (1870-1955), professeur de chimie industrielle à la faculté depuis 1922, cinq autres professeurs titulaires de la faculté des sciences ou professeurs sans chaire y enseignent ${ }^{22}$.

En 1899, Léo Vignon, successeur de Raulin à la faculté des sciences, à l'ECIL, créé l'École française de tannerie (EFT). Une partie des enseignements est en commun avec l'ECIL, ainsi qu'une partie des enseignants ${ }^{23}$.

Il faut noter également la création d'un Institut de chimie industrielle en 1919 au sein de l'université catholique de Lyon ${ }^{24}$, qui mène quelques étudiants jusqu'au diplôme d'ingénieur dans l'entredeux-guerres.

\footnotetext{
${ }^{22}$ Il s'agit de René Locquin (chimie générale minérale) professeur sans chaire de chimie générale; André Job (chimie physique) professeur sans chaire de chimie physique; Jeanne Lemarchands (électrochimie), professeur sans chaire de chimie industrielle ; François Couturier (chimie et industrie agricole), professeur titulaire de chimie agricole; Henri Longchambon (minéralogie), professeur titulaire de minéralogie théorique et appliquée.

${ }^{23}$ La formation dure deux ans.

${ }^{24}$ L'institut est créé par Gaston Lepercq (1860-1920).
} 
Ces différentes institutions témoignent de l'importance de l'enseignement de la chimie à Lyon, ce qui est notable également pour la période du $\mathrm{XIX}^{\mathrm{e}}$ siècle $^{25}$, et pour l'industrie chimique.

Pour ces raisons, le cas de Lyon est intéressant pour étudier l'émergence du titre d'ingénieur-docteur, d'autant plus que le premier titre est délivré en 1926, peut-être même avant Paris et qu'il s'agit, avec Grenoble, pour la période allant jusqu'en 1958 d'une des plus actives villes de province pour ce nouveau diplôme.

\section{Partie 1: Les thèses d'ingénieur-docteur en chimie et leur importance au sein de la faculté des sciences de Lyon}

J'ai identifié, dans le fonds de la Bibliothèque nationale de France, 99 thèses d'ingénieur-docteur soutenues à Lyon jusqu'en $1958^{26}$. Ce chiffre est corroboré par la Revue trimestrielle de l'Association des ingénieurs docteurs de France d'avril, mai, juin 1959 annonçant par sa section lyonnaise la centième thèse soutenue à l'université de Lyon. Il y est également mis en avant l'accélération que connaît le titre, soulignant qu'il s'est écoulé vingt-cinq ans entre la première et la cinquantième thèse et huit ans entre la cinquantième et la centième thèse. Quelques volumes de thèse ont disparu mais,

\footnotetext{
25 Thivend Marianne, «L'enseignement technique et la promotion scolaire et professionnelle sous la Troisième République », Revue française de pédagogie, 159 (avril-juin 2007), en ligne depuis le $1^{\text {er }}$ avril 2011, consulté le 17 mars 2016. URL: http://rfp.revues.org/1045. Caschera Stéphane, L'enseignement de la chimie: l'École de chimie industrielle de Lyon (1876-1939), mémoire de maîtrise en histoire contemporaine, université Lumière-Lyon 2, 1998.

${ }^{26}$ Les thèses de sciences de l'université de Lyon sont cotées en 4-R-1212 $\left(\mathrm{N}^{\circ}\right.$ d'ordre) pour les thèses de doctorat d'État, en 4-R-1212 (Bis, $\mathrm{N}^{\circ} \mathrm{d}$ 'ordre) pour les thèses d'université, et en 4-R-1212 (Ter, $\mathrm{N}^{\circ}$ d'ordre) pour les thèses d'ingénieurdocteur.
} 
pour l'entre-deux-guerres, j'ai pu consulter toutes les thèses qui y ont été soutenues.

Le succès du titre d'ingénieur-docteur est rapide à Lyon, la première thèse est soutenue en 1926 dans les mêmes temps que Paris, c'est-à-dire dès que le titre est mis en place, loin devant Nancy (1932), Toulouse (1937) et Grenoble (1935). Grenoble mis à part, le nombre de thèses d'ingénieur-docteur est aussi significativement plus élevé à Lyon qu'à Nancy (une soixantaine jusqu'en 1958) et Toulouse (25 thèses jusqu'en 1958).

\section{Un titre " trusté " à Lyon par les anciens élèves de l'École de chimie industrielle de Lyon (ECIL)}

La première thèse d'ingénieur-docteur est soutenue en 1926 par Maurice Fluchaire (ECIL, 1921), sur « les condensations par les alcoolates magnésiens mixtes ». Le travail de recherche a été réalisé au laboratoire de chimie générale de la faculté des sciences sous la direction de Victor Grignard. Fluchaire remercie la Société chimique des usines du Rhône qui a financé le travail. Pour autant, ce n'est pas l'entreprise qui a fixé les objectifs des recherches, ceux-ci correspondent pleinement aux axes de recherche de Grignard. Si elle peut y voir un intérêt, toutefois elle n'oriente pas.

La présence d'anciens élèves de l'ECIL dans les thèses d'ingénieur-docteur préparées dans l'entre-deux-guerres est massive. Sur vingt-six thèses, vingt-trois sont le fait d'ingénieurs ECIL. Les trois autres sont préparées par un diplômé de l'Institut de chimie de Paris, promotion $1927^{27}$, un de l'École centrale Lyonnaise, et un

\footnotetext{
${ }^{27}$ Archives de l'École nationale supérieure de chimie de Paris (ENSCP). Dossiers des élèves.
} 
ingénieur des arts et métiers ${ }^{28}$. Pour la période allant jusqu'en 1958, sur les 90 thèses retrouvées sur 99, 77 auteurs sont des anciens élèves de l'ECIL soit $86 \%$. Il faut cependant noter que le nombre d'étudiants préparant une thèse d'ingénieur-docteur à Lyon reste très minoritaire par rapport aux nombres d'ingénieurs diplômés, ce qui apparaît nettement grâce au graphique ci-dessous présentant le nombre d'anciens élèves par année entre 1919 et 1933 :

Tableau $n^{\circ} 1$

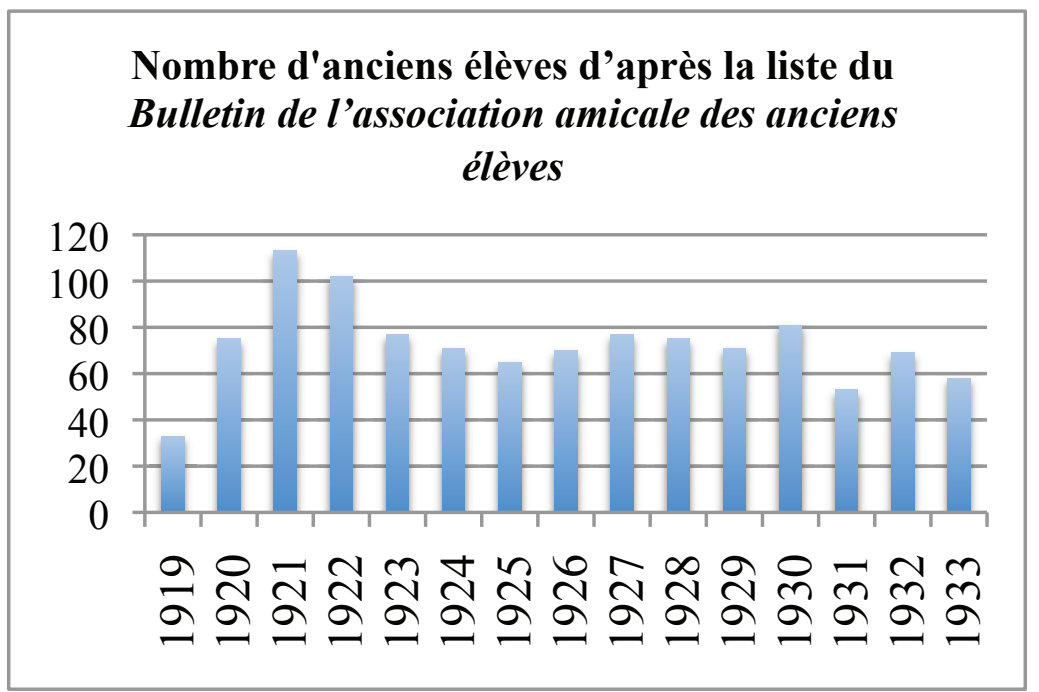

Le début rapide des thèses d'ingénieur-docteur à Lyon s'explique par l'existence de l'École de chimie lyonnaise et de son inscription forte au sein de la faculté des sciences. La très faible présence d'ingénieurs venant de l'École centrale lyonnaise et

\footnotetext{
${ }^{28}$ Par la suite, j'écarterai les deux thèses préparées par l'ingénieur de l'École centrale de Lyon sur «l'aérocinescopie par étincelles », soutenue en 1935, et par l'ingénieur des arts et métiers sur «la télévision, de quelques unes des causes principales de son essor et de ses difficultés de diffusion dans le public jusqu'à nos jours », soutenue en 1934.
} 
l'absence d'étudiants venant de l'Institut de chimie et de physique industrielles vont dans ce sens.

À Lyon, les locaux communs aux deux établissements, ECIL et faculté des sciences, amphithéâtre et laboratoire, et la présence de professeurs communs dont le statut permet d'encadrer des thèses, concourent au démarrage rapide du titre. Il existe en France d'autres écoles de chimie ayant des liens forts avec une faculté des sciences sans que, pour autant, il y ait un essor rapide des thèses d'ingénieurdocteur $^{29}$. Il faut donc voir ici la marque d'une continuité forte entre les deux établissements, en dépit du statut de l'ECIL adopté en 1919, qui, en première lecture, peut être compris comme un éloignement de la faculté des sciences. Cette continuité apparaît par l'étude de l'implantation géographique des établissements, des professeurs et des étudiants en commun aux établissements.

\section{Les thèses d'ingénieur-docteur par rapport aux thèses d'université et aux thèses d'État soutenues en chimie à Lyon pendant l'entre- deux-guerres}

Un état des lieux montre avant tout que la discipline chimie est très bien représentée à Lyon à la fois par le nombre de professeurs et de maîtres de conférences et par le nombre de thèses soutenues. Ainsi, $35 \%$ des thèses d'État et $58 \%$ des thèses d'université dans cette période sont des thèses de chimie. Quant aux professeurs, en 1929, trois titulaires sur treize sont des chimistes (Grignard, chimie générale; Meunier, chimie industrielle ; Couturier, chimie agricole).

\footnotetext{
${ }^{29}$ Fonteneau Virginie, « D'un enseignement de chimie pratique et industrielle à une formation d'ingénieur-chimiste : les débuts de l'Institut de chimie de Paris (18961948) », in L'industrie chimique en question, Lamard Pierre et Stoskopf Nicolas (dir), Éditions Picard, Paris, 2010, p. 33-65.
} 
Et s'il n'y a qu'un maître de conférences sur huit au total, la proportion est de 50\% des professeurs sans chaire, c'est-à-dire trois sur six (Locquin, chimie générale; Job, chimie physique ; Lemarchands, chimie industrielle), ce qui s'explique par ce statut particulier où un complément de salaire doit être apporté par rapport au salaire versé par l'État.

Si l'on s'intéresse à présent à l'importance des thèses d'ingénieur-docteur par rapport aux autres thèses préparées en chimie, il apparaît que leur nombre ne connaît pas d'évolution marquante pendant la période et que les thèses prédominantes sont surtout des thèses d'université, comme le montre le tableau ci-dessous.

Tableau $n^{\circ} 2$



Grâce à la consultation des thèses d'université déposées à la Bibliothèque nationale de France, dont le répertoire croisé avec d'autres ressources permet de supposer que ce recensement est fiable, 
j'ai pu dénombrer 44 thèses d'université soutenues à Lyon pour l'entre-deux-guerres. Les premières thèses d'université à Lyon sont dominées par la chimie comme c'est aussi le cas pour les thèses d'ingénieur-docteur. Ceci n'a rien de surprenant, mais ce qui est inattendu concerne l'origine des étudiants réalisant une thèse d'université. Il s'agit, en effet, ici majoritairement d'ingénieurs : 73\% soit 32 sur 44 thèses. Vingt-cinq ingénieurs ont été formés à Lyon, vingt-et-un sont des ingénieurs diplômés de l'ECIL et quatre sont des diplômés de l'École française de tannerie ou chef de laboratoire de l'École française de tannerie, soit 57\%.

Deux hypothèses peuvent expliquer cette présence. Premièrement, la situation spécifique lyonnaise: en effet, la configuration expliquant le début rapide des thèses d'ingénieurdocteur est déjà là lors de la mise en place du doctorat d'université (hormis l'existence d'une chaire de chimie industrielle à la faculté des sciences). La deuxième possibilité est que le titre de docteur d'université soit investi par des ingénieurs au niveau national ${ }^{30}$. Mais alors une question se pose dans les deux cas : pourquoi créer un titre d'ingénieur-docteur? Il semble que le doctorat d'université ait pu remplir le vide, depuis longtemps mis en évidence, d'une absence de formation de recherche à la différence de ce qui existait en Allemagne.

Pour pousser encore l'analyse, il est nécessaire de s'intéresser aux thèses d'État. Sur trente thèses d'État soutenues à Lyon en chimie dans l'entre-deux-guerres, six sont préparées par des ingénieurs ECIL, ce qui représente tout de même $20 \%$.

\footnotetext{
${ }^{30} \mathrm{Il}$ faudra vérifier cette hypothèse dans des travaux de recherches ultérieurs.
} 
Un résultat important est la mise en évidence d'un afflux d'ingénieurs formés localement non seulement dans les thèses d'ingénieur-docteur, mais aussi d'université et d'État de la faculté des sciences de Lyon. Ceci confirme une place importante d'acteurs différents des étudiants académiques classiques attendus dans les universités et rejoint en ce sens les analyses de Mary Jo Nye. De ce point de vue, il est très possible comme l'affirme cette historienne que certains professeurs ou étudiants davantage inscrits dans le moule académique aient perçu la présence des ingénieurs comme une contamination de la science pure par des sujets de chimie industrielle $\mathrm{e}^{31}$. Selon le point de vue de ces acteurs, cette contamination se traduirait par une baisse de niveau de la faculté des sciences. Rien d'étonnant alors que certains étudiants manifestent le désir d'aller là où se cultive uniquement la science pure.

\section{Partie 2 : Une prédominance des sujets de chimie industrielle au détriment de la chimie pure ?}

L'ambition de cette deuxième partie est d'interroger ces discours en m'intéressant au contenu des thèses d'ingénieur-docteur, d'université et d'État, avec deux axes de réflexion: ces thèses réalisées par les ingénieurs portent-elles toutes sur des sujets de chimie industrielle? Les thèses de chimie pure portent-elles trace de la présence des ingénieurs? En d'autres termes, la chimie industrielle

\footnotetext{
${ }^{31}$ Voir Nye M.J., Science in the Provinces, op. cit. note 4, p. 233. M. J. Nye explique qu'il ne faut pas chercher la raison de ces affirmations dans les institutions françaises car on retrouve le même discours en Angleterre et en Allemagne. Les termes science pure, chimie pure, science appliquée, utilisés à cette époque, sont repris dans cet article.
} 
submerge-t-elle la chimie pure ou la présence importante des ingénieurs nourrit-elle également la recherche en chimie pure?

\section{Des thèses d'ingénieur-docteur non orientées systématiquement vers l'industrie}

Les thèses d'ingénieur-docteur de l'entre-deux-guerres à Lyon s'inscrivent dans deux laboratoires, celui de Grignard en chimie générale et celui de Meunier en chimie industrielle. Autant de thèses d'ingénieur-docteur sont préparées dans le laboratoire de Grignard jusqu'en 1935 qu'au laboratoire de Meunier. En revanche, il est très net qu'après le décès de Grignard, son successeur Locquin n'encadre que très peu de ces thèses par rapport à Meunier.

Les tableaux (3-6) ci-dessous donnent la liste des thèses en fonction du laboratoire où elles sont préparées et en fonction des financements. Ces thèses sont presque toutes dirigées par Grignard et par Meunier. Pour certaines thèses, il apparaît que Meunier n'a pas été l'initiateur ou le directeur au quotidien mais ce n'est pas toujours clair. Deux thèses d'ingénieur-docteur se démarquent, l'une préparée au laboratoire de chimie biologique de Claude Fromageot (1899-1958), professeur sans chaire, et l'autre préparée au laboratoire de l'École française de tannerie.

\section{Tableau $\mathbf{n}^{\circ} \mathbf{3}$}

Thèses préparées au laboratoire de chimie générale de la faculté des sciences dirigé par Grignard (7) puis Locquin à partir de 1936 :

Société chimique des usines du Rhône:

1926 : Maurice Fluchaire, Condensations par les alcoolates magnésiens mixtes.

Société des matières colorantes et produits chimiques de Saint-Denis :

1927 : Georges Mingasson, La catalyse dans le vide.

1930 : Henri Delarue, Contribution à l'étude des énols. 
Société des usines chimiques Rhône-Poulenc ${ }^{32}$ (prêt d'honneur) :

1929 : Pierre Velon, Hydrogénation catalytique sous pression réduite.

Carte des nuances de la Fédération de la soie :

1932 : André Fargier, Hydrogénation catalytique sous pression réduite.

Urbain Corporation :

1928 : Gabriel Simonin, Contribution à l'étude des charbons actifs.

Sans financement :

1934 : Georges Colonge, Recherches sur la préparation des cétols et des cétones éthyléniques au moyen des composés aminomagnésiens mixtes.

1936 (Locquin) : Robert Donzallaz, Pyrolyse des sels de nicotine existant dans le tabac.

Les titres ainsi que le contenu des thèses mettent en évidence que seules quinze sur vingt-quatre portent sur des sujets de chimie industrielle. Huit portent sur la chimie générale organique dont sept ont été dirigées par Grignard et une par Fromageot. Enfin, il faut mentionner la première thèse dirigée par Locquin qui n'est ni une thèse de chimie générale, ni une thèse de chimie industrielle. Donzallaz $^{33}$ est diplômé en criminalistique, ancien assistant du Dr Locard au laboratoire de police scientifique de Lyon. Sa thèse porte sur la détermination des types de tabac par pyrolyse.

\section{Tableau n ${ }^{\circ} 4$}

\section{Thèses préparées au laboratoire de chimie industrielle de la faculté des sciences} dirigé par Meunier :

Carte des nuances de la Fédération de la soie:

1927 : Georges Rey, Contribution à l'étude de la laine.

1929 : Raymond Guyot, Contribution à l'étude des soies artificielles.

1933 : Gonfard Maurice, Contribution à l'étude des propriétés de la benzylcellulose.

32 J'ai gardé le nom de l'entreprise tel qu'il a été transcrit dans la thèse.

${ }^{33}$ Les acteurs, dont il est question dans cet article, sont peu connus et peu visibles. Dans le cadre de cette recherche, je n'ai pas pu faire de recherche précise sur leur état-civil. 
Chambre syndicale de la grande industrie chimique :

1939 : Jean Vessereau, Recherches sur l'électrolyse ignée. Cas des oxydes dissous dans les phosphates fondus.

Compagnie française de produits chimiques et matières colorantes de Saint-Clair du Rhône :

1929 : Fred Livet, 1) contribution à l'étude de l'action des électrolytes dans la teinture du coton par les colorants substantifs. 2) les colorants pour cuve.

Fondation scientifique de Lyon et du Sud-Est:

1928 : Louis Michallet, Contribution à l'étude de l'oxydation des huiles de poissons et application au chamoisage.

Comité des industries chimiques de France : Prêt d'honneur

1935: Pierre Audry, Contribution à l'étude des propriétés de l'osséine et de la fabrication de la colle d'os.

Sans financement :

1931 : Jacques Corbière, Sur la fixation des matières grasses émulsionnées par les fibres textiles.

1937: André Tardy, Action du formol sur les fonctions alcooliques de quelques glucides.

1937 : Georges Vayssière, Contribution à l'étude des résines vinyliques.

1937: Ernest Charles, Contribution à l'étude de la concentration des solutions aqueuses d'acide acétique en présence d'un tiers corps. Application au pyroligneux. 1938: Georges Bost, Contribution à l'étude de la fermentation propionique. Application à la fabrication de l'acide propionique.

1938: Kemal Mostafavi, Contribution à l'étude de certaines réactions de condensation utilisant les chlorures d'acides en présence de chlorures métalliques.

1939 : Grigorij Slovsky, Contribution à l'étude des résines méthacryliques.

Tableau $\mathbf{n}^{\circ} \mathbf{5}$

Thèses préparées au laboratoire de chimie biologique de la faculté des sciences dirigé par Fromageot :

Sans financement:

1939: Paul Heitz, Nouvelles méthodes de dosage spécifique de quelques acides aminés : alanine, valine, leucine, sérine et acide aspartique.

Tableau $n^{\circ} 6$

Thèses préparées au laboratoire de recherches de l'École française de tannerie dirigé par Meunier :

Sans financement :

1938 : Jean-Noël Duperray, Fermentation citrique. 
Les sujets de thèse relevant de la chimie industrielle portent sur l'étude de matériaux (laine, cuir, soies artificielles), du travail du textile (colorants, ensimage/encollage), des procédés (acide pyroligneux), de polymères et matières plastiques (résines vinyliques, résines méthacryliques, benzyl cellulose), fabrication d'acides utiles notamment dans les synthèses d'intérêt industriel (acide citrique, acide propionique). Il y a également une thèse sur les colles à base de poisson (Meunier avait créé un service dans son laboratoire de chimie à la demande de fabricants de colle). Toutes ces thèses sont préparées au laboratoire de Meunier.

Les thèses d'ingénieur-docteur sont donc majoritairement tournées vers des sujets de chimie industrielle mais pas uniquement. Les sujets préparés dans le laboratoire de Grignard sont caractéristiques de ses axes de recherches, ici des recherches sur la condensation et l'énolisation possible avec les organomagnésiens ${ }^{34}$ (Fluchaire, Delarue et Collonge), et des thèses sur l'hydrogénation catalytique sous pression réduite (Mingasson, Vélon et Fargier).

Si l'on s'intéresse maintenant aux financements venant de l'industrie, treize thèses au total sont subventionnées à des niveaux divers (financement de la thèse, prêt de matériel, aide pour l'impression de la thèse, etc.). Ces subventions ne sont pas orientées préférentiellement vers des sujets de chimie industrielle, il y a autant

\footnotetext{
${ }^{34}$ Pour pousser à bout cette analyse, il faudrait prendre pour objet les étudiants de Grignard sur l'ensemble de la période et étudier finement les sujets de recherches et les liens entre les différentes thèses réalisées au sein du laboratoire de chimie générale.
} 
de thèses financées en chimie générale ${ }^{35}$. De ceci, il en résulte que l'intérêt des industries pour la recherche ne se traduit pas systématiquement par un pilotage vers des sujets industriels, ni directement en rapport avec l'entreprise qui finance.

Cette analyse montre qu'une thèse d'ingénieur-docteur malgré un contexte de création très marqué par l'industrie n'est pas systématiquement orientée vers l'industrie, comme nous avons pu le voir avec les thèses dirigées par Grignard, financées autant que les thèses de chimie industrielle.

\section{La part de la chimie industrielle dans les thèses d'université}

La présence importante des ingénieurs dans les thèses d'université s'impose encore plus quand on ne prend en compte que les sujets de chimie industrielle. Il est remarquable d'y constater la part prise par des sujets concernant l'industrie du cuir, ce qui n'était pas le cas des thèses d'ingénieur-docteur. Onze thèses dont la première est soutenue en 1924 ont pour sujet le cuir ${ }^{36}$. La première thèse d'université, soutenue par Paul Chambard, ingénieur ECIL et professeur à l'École française de tannerie, est un cas intéressant à plus d'un titre. Tout d'abord, il s'agit d'une thèse préparée sous la direction de Meunier titularisé dans sa chaire de chimie industrielle en 1922. Celui-ci est alors également directeur-adjoint de l'ECIL et directeur de

\footnotetext{
${ }^{35}$ Une étude poussée des financements de thèse par les industriels doit être menée pour affiner ces conclusions.

${ }^{36}$ Les auteurs de ces thèses sont Paul Chambard, Ernest Mezey, Anthelme Jamet, Charles Gastellu, LeViet Khoa, Jean Azémar, E.-M Schweikert, Reginald Garnot, Marcel Seux, Auguste Boutin, Ahmed Abassi. L'intérêt du Syndicat des cuirs et peaux pour la recherche n'a pas encore fait l'objet d'études historiques, pas plus sur les thèses réalisées, que sur l'impact de ces recherches sur l'activité du cuir.
} 
l'EFT depuis 1921. C'est le Syndicat des cuirs et peaux qui finance un laboratoire de recherche à l'EFT. Par la suite, Chambard devient sousdirecteur de l'EFT, puis directeur, ainsi que président de l'Union internationale des sociétés des chimistes des industries du cuir. Les sujets de ces thèses portent majoritairement sur le tannage du cuir, et également une sur les colorants chromés, une sur l'épilage des peaux et une sur le salage des peaux.

Les autres sujets de chimie industrielle sont variés, deux thèses portent sur les colorants, mais il n'apparaît pas d'autre thème structurant comme l'est celui du cuir ${ }^{37}$.

Outre ces thèses de chimie industrielle préparées par des ingénieurs, trois thèses d'université sont soutenues en criminalistique (Harry Soderman, Alfred Carrel, Jacques Locard) ${ }^{38}$.

Les ingénieurs ne préparent pas seulement des thèses d'université dans des thématiques de chimie industrielle. En effet, sur quatorze thèses d'université soutenues avec Grignard comme président de jury, onze sont le fait d'ingénieurs dont dix viennent de l'ECIL. Deux d'entre eux sont à considérer à part : Louis Burtin, dont la thèse, «Contribution à l'étude des équilibres entre les chlorures et les bromures alcalins en solution », a été préparée sous la direction d'André Job, et dont le sujet n'entre pas dans les axes de recherches

\footnotetext{
${ }^{37}$ Il faudrait néanmoins ici aussi faire un travail d'analyse approfondi impossible dans le cadre de cet article.

${ }^{38}$ Soderman Harry, Recherches pour l'expertise des armes à feu courtes, 1928 ; Carrel Alfred, Contribution à l'identification des encres en criminalistique, 1934 ; Locard Jacques, Contribution à l'étude de l'oxydation du graphite en milieu alcalin, 1936. Cette thématique particulière nécessiterait un travail de recherche spécifique incluant la thèse d'ingénieur-docteur de Donzallaz.
} 
de Grignard ; Tamisier, dont la thèse «Recherches sur la constitution de quelques amines ", a été dirigé par Auméras qui devient quelques années plus tard professeur de chimie physique à la faculté des sciences de Lyon. Deux thèses d'université sont également à traiter un peu à part mais sont proches ou dans les thématiques de Victor Grignard : Michelet dont la thèse «Études de quelques aldéhydes $\alpha$ alcoylcinnamiques simples et substitués » a été préparée non pas à la faculté des sciences mais dans les laboratoires des Établissements Descollonges frères ; celle de Louis Leers, «Étude de transpositions pinacoliques dans la série des triméthyl-alcoylglycols » dirigée par Locquin, qui reprend à Lyon la chaire de chimie générale après le décès de Grignard.

Les autres ingénieurs, Escourrou, Dubien, Perrichon, Chambret, Jean Colonges, Lapayre, Blanchon, travaillent sous la direction de Grignard sur ses axes de recherches: a) les organomagnésiens utilisés pour leur propriété énolisante étudiée par Blanchon, «Phénomènes d'énolisation. Recherches sur la préparation des énols et leurs préparations », et pour leur capacité de condensation par Dubien, «Recherches sur les dérivés obtenus par l'action des composés organomagnésiens sur la butylidène-acétone et sur son cétol» ainsi que Colonges, «Contribution à l'étude de la condensation des cétones $»$; b) La chimie des terpènes, et en particulier la pulégone (thèse d'université d'Escourrou sur la méthylhepténone naturelle); c) les alcools tertiaires, thèse de Chambret sur la stabilité des alcools tertiaires. Les thèses de Perrichon et Lapayre portent quant à elles plus spécifiquement sur les carbures acétyléniques. 
Les entreprises qui financent les thèses d'université sont les mêmes que celles qui financent les thèses d'ingénieur-docteur. La répartition est à peu près identique, sept thèses d'université sont financées sous la direction de Meunier et six sous la direction de Grignard $^{39}$. Les mêmes conclusions peuvent donc être tirées quant au non pilotage systématique par les entreprises des sujets de recherches de chimie générale.

Avec cette analyse, il faut souligner que les ingénieurs s'investissent dans la recherche également en chimie générale et non pas uniquement en chimie industrielle. Ce bassin d'ingénieurs est ainsi un apport humain aux laboratoires de recherches de la faculté des sciences, où ils contribuent aussi à la recherche en science «pure ». De même, l'autre lien visible avec l'industrie, les financements de thèse, alimentent tout autant des recherches intéressées que des recherches de chimie générale. C'est donc tout à la fois la vie de la recherche en chimie générale et en chimie industrielle qui est stimulée par cette présence d'ingénieurs et les liens avec l'industrie.

\section{La part de la chimie industrielle dans les thèses d'État}

Comme je l'ai précisé, le doctorat d'université ne donne pas plus accès à des carrières universitaires que la thèse d'ingénieur-

\footnotetext{
${ }^{39}$ Pour Grignard, il s'agit de la Société chimique des usines du Rhône $(1,1924)$, de la Compagnie nationale des matières colorantes $(2,1925 ; 1,1930)$, de la Société des matières colorantes et produits chimiques de Saint-Denis $(1,1925)$ et de Kuhlmann (1, 1930). Pour Meunier, les financements sont donnés par le Syndicat des cuirs et peaux de France $(1,1924)$ par la Fondation scientifique de Lyon et du sud-est et la Direction des recherches scientifiques et industrielles et des inventions $(1,1924)$, de la Carte des nuances de la Fédération de la soie $(1,1927 ; 1,1928)$, de la Société d'encouragement pour l'industrie nationale $(1,1928)$, du Comptoir des textiles artificiels $(1,1932)$, de la Compagnie française de produits chimiques et matières colorantes de Saint-Clair du Rhône (1, 1933).
} 
docteur. Pour notre démonstration, il reste alors à examiner les thèses d'État.

La part des ingénieurs préparant des thèses d'État s'élève à $20 \%$ ce qui est loin d'être négligeable. Sur les six ingénieurs s'engageant dans une thèse d'État, je n'ai pas retrouvé trace de Tchao Yin Lai ${ }^{40}$, ingénieur chimiste, qui fait des études à Lyon dans le cadre de l'Institut franco-chinois de Lyon. Les trajectoires des cinq autres sont très significatives. Trois d'entre eux ont fait une carrière académique.

Jean Savard ${ }^{41}$, ingénieur ECIL, bénéficie de deux bourses Rockefeller après sa thèse, une pour un séjour de 12 mois en 1927 au laboratoire du Professeur Henri à l'École polytechnique de Zurich, une pour 4 mois en Suède en $1933^{42}$. Il devient professeur de chimie générale à la faculté des sciences de Lille à partir de 1944.

Michel Lesbre (1908-1999) ${ }^{43}$, ingénieur ECIL, obtient sa thèse d'État en 1931 puis part travailler au laboratoire de Job comme boursier. Chargé de cours à la faculté des sciences de Besançon puis à Lyon, il est nommé maître de conférences en 1949 à Toulouse, où il termine sa carrière comme professeur titulaire de la chaire de chimie organique. Il fonde en 1954 à Toulouse le laboratoire des

\footnotetext{
${ }^{40}$ Tchao Yin Lai, Étude sur les éthers bromhydriques des alcools primaires $\alpha$ acétyléniques, doctorat ès sciences physiques, université de Lyon, 1932.

${ }^{41}$ Jean Savard, La pulégone - son énolisation - ses dérivés, doctorat ès sciences physiques, université de Lyon, 1926.

${ }^{42}$ Archives Institut Pasteur.

${ }^{43}$ Lesbre Michel, Contribution à l'étude des solutions de colorants substitutifs, doctorat ès sciences physiques, université de Lyon, 1931.
} 
organométalliques, spécialisé dans la recherche sur les dérivés du germanium $^{44}$.

Pierre Desnuelle suit lui aussi un parcours académique du côté de la chimie biologie, il est assistant à la faculté des sciences de Lyon en 1936, maître de conférence de chimie biologique à Marseille en 1943, puis professeur en 1947.

Deux élèves ont des trajectoires liées à l'industrie bien que leurs thèses ne portent pas sur des sujets industriels.

René Escourrou ${ }^{45}$ travaille un temps au sein des Papeteries Navarre, dont le fondateur, André Navarre, participe à la création de l'École française de papeterie en 1908, où il y enseigne un temps. Il est l'auteur d'un ouvrage de références sur la fabrication du papier, plusieurs fois réédités ${ }^{46}$.

Walter Juda ${ }^{47}$, né à Berlin, a quitté l'Allemagne en 1933 en raison de la montée du parti nazi. Après un séjour en Suisse, comme réfugié, il est arrivé en France et a suivi le cursus de l'École de chimie industrielle de Lyon, puis a fait une thèse dont le sujet non orienté vers la chimie industrielle a été suggéré par Lemarchands. Il travaille sur la question de la surtension de l'hydrogène sur des cathodes métalliques. Il parvient en 1939 à obtenir un visa étudiant pour partir

\footnotetext{
${ }^{44}$ Satgé Jacques, « Michel Lesbre (1908-1999), nécrologie », L'Actualité chimique (août-septembre 1999), p. 38.

${ }^{45}$ Escourrou René, Hydrogénation catalytique dans le vide, doctorat ès sciences physiques, université de Lyon, 1925.

${ }^{46}$ Escourrou R., Le papier, Armand Colin, Paris, 1941. $2^{\text {ème }}$ édition en 1948. Il existe une autre édition en 1956. Un nouveau livre paraît en 1958, intitulé $L a$ fabrication du papier, toujours chez Armand Colin.

${ }^{47}$ Juda Walter, Recherches sur la surtension de l'hydrogène et ses causes », doctorat ès sciences physiques, université de Lyon, 1939.
} 
aux États-Unis. Il travaille au Harvard Chemistry Department de 1939 à 1948. Il rejoint ensuite le Massachussets Institute of Technology. Il cofonde une première entreprise pour la désalinisation de l'eau, en 1948, Ionics Inc., une seconde, sur les piles à combustible, en 1961, Prototech Co, et la troisième en 1992, Hy9, qui développe une technologie concernant la production et la purification de l'hydrogène ${ }^{48}$.

Encore une fois, les ingénieurs ne s'orientent pas systématiquement vers des sujets ou des trajectoires industriels. De manière similaire, un étudiant au parcours académique ne poursuit pas systématiquement des recherches de science pure. Ainsi trois thèses préparées par des non ingénieurs ont des intérêts industriels revendiqués.

Léonce Bert ${ }^{49}$, professeur au lycée Blaise Pascal de ClermontFerrand et chef de travaux en chimie industrielle, travaille sur l'édification de la série paracyménique à partir de l'alcool isopropylique. Il soutient sa thèse en 1925. Un des enjeux énoncés par Léonce Bert est de chercher des applications chimiques à l'alcool isopropylique dont le prix était élevé mais qui, fabriqué à partir du cracking, est devenu beaucoup plus abordable et est donc entré dans la pratique industrielle.

Reginald Killmaster, canadien, soutient une thèse en 1924 qui a pour objet l'étude du mécanisme du cracking avec l'action du

\footnotetext{
${ }^{48} \mathrm{http}$ //engineering.tufts.edu/chbe/news/2011/JudaDeath.htm

${ }^{49}$ Léonce Bert est directeur de l'Institut de chimie et de technologies industrielles de Clermont-Ferrand (ICTIC) de 1929 à 1941 et y fonde une section caoutchouc en 1935.
} 
chlorure d'aluminium sur trois hydrocarbures aromatiques ${ }^{50}$. Il fonde en 1924, l'Imperial Oil's Sarnia Research Center, et crée un nouveau process à la raffinerie en utilisant le catalyseur sous forme de poudre ${ }^{51}$. Abel Caille, agrégé de l'université, soutient sa thèse en 1933 sur les éthers cellulosiques, important notamment pour la fabrication des vernis d'aviation ${ }^{52}$.

L'étude des contenus des thèses d'État ${ }^{53}$ et des trajectoires de leurs auteurs, qu'il faudrait approfondir, confirme les résultats obtenus pour l'étude des thèses d'ingénieur-docteur et les thèses d'université. La présence d'ingénieurs ne dirige pas préférentiellement les sujets de thèse vers la chimie industrielle. Les ingénieurs font tout autant que les autres étudiants des thèses de chimie pure et ont également des carrières académiques.

\section{Des recherches d'ingénieur en chimie industrielle et chimie pure}

En matière de bilan, je reprendrai deux exemples liés aux recherches de Grignard. Tout d'abord, pour les recherches sur les

\footnotetext{
${ }^{50}$ Killmaster Reginald, Action du chlorure d'aluminium sur les hydrocarbures aromatiques et aliphatiques saturés, doctorat ès sciences physiques, université de Lyon, 1924.

${ }^{51}$ https://www.highbeam.com/doc/1G1-84806692.html.

${ }^{52}$ Caille Abel, Contribution à l'étude des éthers cellulosiques, doctorat ès sciences physiques, université de Lyon, 1933.

53 Je n'ai examiné ici qu'une partie des thèses d'État. Toutefois, lors de cette analyse, j'ai noté la présence d'autres thématiques qu'il faudrait travailler: premièrement la question des femmes moins présentes que dans les thèses d'université et d'ingénieur-docteur: Jeanne Lemarchands, Paulette Berthier, Suzanne Thévenet, Denise Saunier; deuxièmement la présence d'étudiants venant de l'étranger, Thomas N. Iliesco, Vasile Th. Cherchez, Reginald Killmaster, Sung Wouseng, Walter Juda et un groupe d'étudiants chinois, Tcheou Fa-Khi, Loui Oui Tao, Tchao Yin Lai.
} 
organomagnésiens, j'ai identifié sur ce sujet deux thèses d'ingénieurdocteur, cinq thèses d'université, trois thèses d'État. Sur ces dix thèses, il y a sept ingénieurs dont six viennent de l'ECIL. Ensuite, concernant la catalyse, il y a trois thèses d'ingénieur-docteur et une thèse d'État, les quatre sont préparées par des ingénieurs venant de l'ECIL. Il est ainsi manifeste que la présence d'ingénieurs n’oriente pas nécessairement vers la science industrielle mais que ces ingénieurs contribuent également à des recherches de science. Ceci a une implication méthodologique marquée: il faut, pour l'entre-deuxguerres en France, inclure dans les études sur la recherche en chimie cette catégorie d'acteurs, ainsi que leur formation. Cela a aussi des conséquences sur la construction de l'historiographie.

J'ai montré également que les financements venant de l'industrie sont répartis autant vers la chimie générale que vers la chimie industrielle.

Dans le cas lyonnais, cette étude fait également apparaître que les deux professeurs référents pour les thèses sont Grignard et Meunier. La disparition de Grignard engendre une baisse du nombre d'étudiants s'inscrivant dans des thèses de chimie pure, le relais n'est pas tout de suite pris par ses successeurs. Meunier, quant à lui, reste actif en chimie industrielle. L'importance de la chimie industrielle au milieu des années 1930 n'est pas qu'une question de conquête de territoire, mais tient également à la perte du prix Nobel de chimie, Victor Grignard, qui laisse un vide à la faculté des sciences de Lyon. 


\section{Conclusion}

L'étude de la recherche en chimie à Lyon pendant l'entredeux-guerres par les thèses d'ingénieur-docteur permet de tirer des conclusions à deux niveaux.

Tout d'abord, le cadre d'intelligibilité de la recherche en chimie à Lyon dans l'entre-deux-guerres ne peut être limité à la seule faculté des sciences. Comme je l'ai montré, il doit aussi inclure deux écoles, l'ECIL et l'EFT, dont l'examen du seul statut les distingue de l'université. Une approche méthodologique d'offre locale d'enseignement et de recherche met en évidence la continuité entre ces institutions par des professeurs communs, et le parcours des élèves et, ainsi, donne des clés pour la compréhension d'une institution de statut national: la faculté des sciences. Le fait que les élèves de l'ECIL choisissent très rapidement et quasi exclusivement le titre d'ingénieur-docteur, créé pour faire se rencontrer le monde des ingénieurs et le monde académique, matérialise ces liens entre institutions. Cependant, les ingénieurs de l'ECIL n'ont pas attendu la création de ce titre pour faire de la recherche dans les laboratoires de la faculté des sciences comme je l'ai montré avec l'étude des thèses d'université.

Ces liens forts entre science et industrie, déjà montrés dans l'historiographie, ont été analysés pour l'instant comme une irruption de l'industrie dans la science pure, industrie qui deviendrait dominante dans l'entre-deux-guerres. Cette analyse repose sur une idée implicite: un ingénieur qui fait de la recherche en chimie se tourne spécifiquement vers la chimie industrielle. Or en analysant les contenus de thèse, j'ai montré que les ingénieurs ne s'orientent pas 
exclusivement vers la recherche industrielle, que ce soit pour le titre d'ingénieur-docteur, le doctorat d'université ou pour les thèses d'État. Il y a plusieurs conséquences à cela, la première est que les ingénieurs ont augmenté le potentiel de recherche en chimie pure et pas uniquement en chimie industrielle ${ }^{54}$.

Le second point concerne les sources utilisées pour étudier la recherche. Ne prendre que les thèses d'État et d'université pour étudier la recherche qui se fait dans l'entre-deux-guerres constitue un biais fort que ce soit pour les sujets de science appliquée ou de science pure.

Enfin, cet article déconstruit l'idée d'institutions académiques séparées d'institutions d'enseignement technique supérieur, de parcours normés au sein des facultés des sciences. C'est la diversité des parcours et des trajectoires qui ressort ici, de l'ingénieur soutenant une thèse d'État pour embrasser une carrière académique, à l'ingénieur menant des recherches de chimie industrielle traçant sa voix dans une carrière industrielle, aux étudiants académiques menant des travaux intéressant la chimie industrielle et l'industrie pour ensuite mener carrière académique ou industrielle. Cet article appelle à reconsidérer les frontières implicitement admises entre deux pôles science/université et ingénieur/industrie, et tout particulièrement, la présence et le parcours des ingénieurs diplômés au sein de l'université.

\footnotetext{
${ }^{54}$ Mon point de vue n'est pas de dire qu'une thèse de chimie industrielle ne puisse pas apporter une contribution à la chimie générale ou inversement, mais que l'origine « ingénieur » des étudiants ne pilote pas leur inscription dans telle ou telle partie de la chimie lors de poursuite d'études en recherche.
} 